\title{
Medical Image of the Week: Subarachnoid Hemorrhage
}

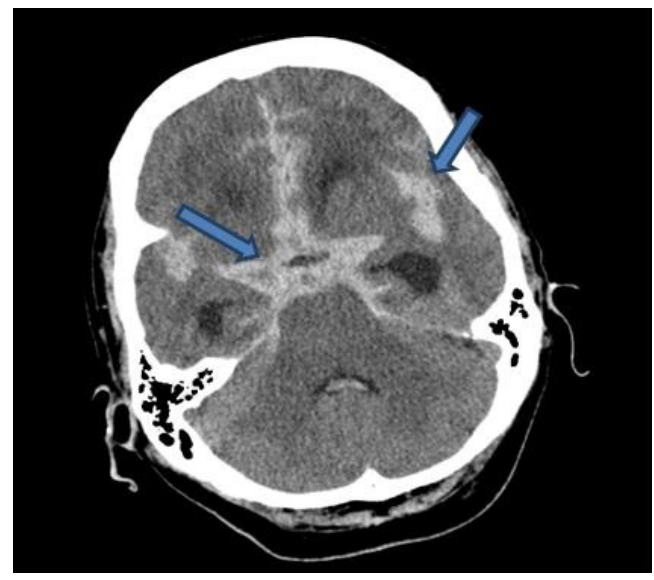

Figure 1. Axial CT of the head without contrast demonstrates a large subarachnoid hemorrhage in the basal cisterns and adjacent to the insular cortices (blue arrows).

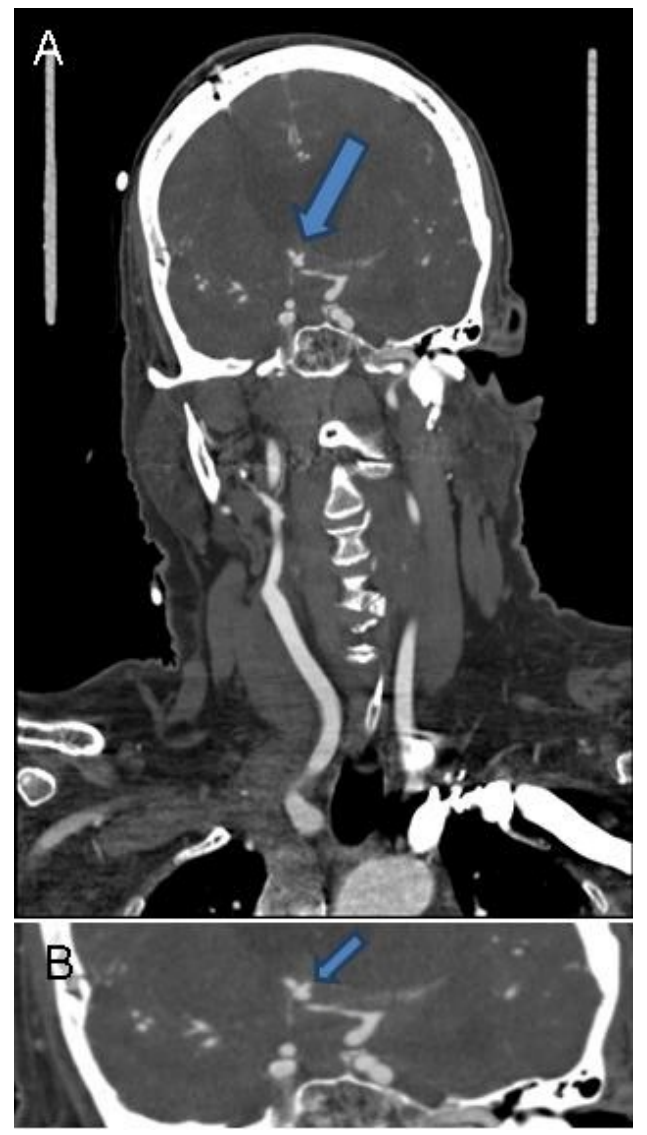

Figure 2. Coronal CT angiogram of the head demonstrates a saccular outpouching of the anterior communicating artery (blue arrow) consistent with an aneurysm. 
A 70-year-old lady with a past medical history of hypertension and dyslipidemia was brought in by her family members for evaluation of confusion and headache for 1 week. There was no history of recent trauma or falls. There was no known family history of aneurysm or sudden death. On examination, her blood pressure was 139/99 $\mathrm{mmHg}$, heart rate $92 \mathrm{bpm}$, afebrile and respiratory rate was 13 breaths per minute. She was alert but only oriented to self. Pupils were symmetric and reactive to light. She was able to follow commands and power was symmetric in all limbs.

CT of the head without contrast showed diffuse subarachnoid and intraventricular hemorrhage with signs of raised intracranial pressure (Figure 1). Neurosurgery was consulted and she underwent emergent insertion of an external ventricular drain. Head CT post-ventriculostomy showed improvement in her ventricular dilatation. CT angiography was performed later and showed an anterior communicating artery aneurysm (Figure 2), thought to be culprit of her subarachnoid hemorrhage. Craniotomy with surgical clipping was performed. This was followed by improvement in her mental status.

The common presenting symptom of patients with subarachnoid hemorrhage is headache. They will classically describe it as "worst headache of my life". This can be accompanied by altered mental status, nausea, vomiting, or meningeal signs. Head CT without contrast should be obtained immediately if there is suspicion of subarachnoid hemorrhage. Studies have shown that head CT is extremely sensitive if obtained within 6 hours of clinical presentation but its sensitivity declines over time (1). Lumbar puncture should be performed if head CT is negative but there is strong suspicion of subarachnoid hemorrhage. A combination of negative head CT and lumbar puncture is sufficient to rule out subarachnoid hemorrhage in a patient presented with headache (2).

Kai Rou Tey ${ }^{1}, \mathrm{MD}$; Tammer Elaini ${ }^{2}, \mathrm{MD}$

${ }^{1}$ Department of Internal Medicine, University of Arizona College of Medicine- South Campus and ${ }^{2}$ Department of Pulmonary, Critical Care, Allergy and Sleep University of Arizona College of Medicine

Tucson, AZ USA

\section{References}

1. Perry JJ, Stiell IG, Sivilotti ML, et al. Sensitivity of computed tomography performed within six hours of onset of headache for diagnosis of subarachnoid haemorrhage: prospective cohort study. BMJ. 2011;343:d4277. [CrossRef] [PubMed]

2. Perry JJ, Spacek A, Forbes M, et al. Is the combination of negative computed tomography result and negative lumbar puncture result sufficient to rule out subarachnoid hemorrhage? Ann Emerg Med. 2008 Jun;51(6):707-13. [CrossRef] [PubMed] 